\title{
Repercussions of a sleep medicine outreach program
}

\section{S.G. Conway ${ }^{1}$, S. Tufik ${ }^{1}$, \\ R. Frussa Filho ${ }^{2}$ and L.R.A. Bittencourt ${ }^{1}$}

Departamento de Psicobiologia, ${ }^{2}$ Departamento de Farmacologia, Universidade Federal de São Paulo, São Paulo, SP, Brasil

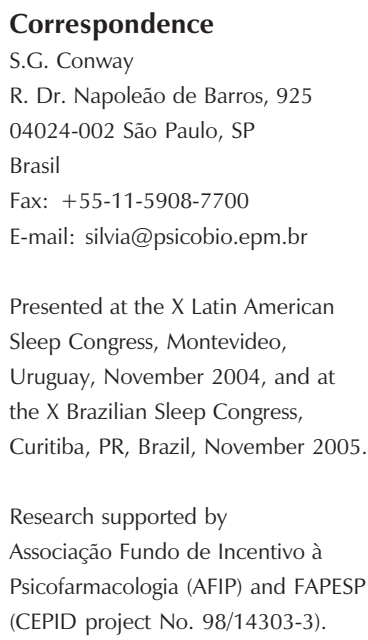

Correspondence

S.G. Conway

R. Dr. Napoleão de Barros, 925

04024-002 São Paulo, SP

Brasil

Fax: +55-11-5908-7700

E-mail: silvia@psicobio.epm.br

Presented at the $\mathrm{X}$ Latin American

Sleep Congress, Montevideo,

Uruguay, November 2004, and at

the $\mathrm{X}$ Brazilian Sleep Congress,

Curitiba, PR, Brazil, November 2005.

Research supported by

Associação Fundo de Incentivo à

Psicofarmacologia (AFIP) and FAPESP

(CEPID project No. 98/14303-3).

Received October 27, 2005 Accepted April 24, 2006

\begin{abstract}
Despite the high prevalence of sleep disorders, many healthcare professionals and lay people have little knowledge of Sleep Medicine. Mindful of such a reality, in 2001 the Sleep Institute of the Associação Fundo de Incentivo à Psicofarmacologia launched a campaign to increase Sleep Medicine awareness. Media features, exhibitions, inserts, and classes were used to reach 2,000,000 people and 55,000 healthcare professionals during the period from 2001 to 2004. To evaluate this program, we compared data for polysomnography referrals to the Institute in 2000 and in 2004. A total of 8805 referrals were evaluated (2000: 2164; 2004: 6641). Over the 4 years of the program, the number of beds increased by $43 \%$; more women were referred ( 31 vs $37 \%$; $\mathrm{P}<0.001$ ), mainly with a diagnostic hypothesis of sleepdisorder breathing (SDB). SDB was the most frequent diagnostic hypothesis in 2000 and 2004. In 2004 there were fewer referrals without a diagnostic hypothesis (27 vs 21\%; $\mathrm{P}<0.001)$ and for controlling surgically treated SDB $(2.3 v s 1.6 \%$; $\mathrm{P}<0.05)$, and an increase in the following diagnostic hypotheses: non-invasive treatment of SDB (8.3 vs $12.3 \% ; \mathrm{P}<0.001)$ and insomnia (3.5 vs 6.5\%; P $<0.001)$. Insomnia diagnostic hypothesis was better correlated with SDB on referral documents in 2004 and less with a diagnostic hypothesis of limb movement disturbance. The program helped increase polysomnography referrals, particularly among women. Healthcare professionals appear to have a more developed understanding of sleep disorders.
\end{abstract}

\section{Introduction}

Sleep disorders are prevalent and affect approximately 70 million people in the United States (1-3). They represent a major risk for public health since sleep disorders are related to respiratory, cardiovascular and neurological problems and higher risks of automobile or workplace accidents $(4,5)$. In a 1996 survey, approximately $81 \%$ of the adult
Key words

- Sleep

- Medical education

- Clinical diagnosis

- Diagnosis-related groups

- Sleep-disorder breathing

- Insomnia population of the city of São Paulo reported sleep complaints, mainly insomnia or snoring (35 and 23\%, respectively) (6). In the middle-aged population of the United States, the estimated prevalence of obstructive sleep apnea-hypopnea syndrome (OSAHS) is $2 \%$ for women and $4 \%$ for men (1).

Retrospective studies demonstrate that sleep disorders are under-recognized, with most cases remaining untreated $(4,7,8)$. Recent surveys 
have shown that formal education in Sleep Medicine for medical undergraduates is rare or non-existing in Brazil and internationally (9-16). Such a program, we feel, badly needs to be created and delivered.

Centers in different countries have sought to fill this gap through outreach programs $(17,18)$. In November 2000, the Sleep Institute (Instituto do Sono), of the Psychopharmacology Support Fund Association (local acronym AFIP), was designated as a Center of Research, Innovation, and Dissemination (local acronym CEPID) with support from the State of São Paulo Research Support Foundation (local acronym FAPESP). The goal of FAPESP support was to develop the Sleep Institute as a center for Sleep Medicine research, technological innovation, and dissemination in São Paulo State. The Sleep Medicine outreach program was developed at the Sleep Institute and the target population selected was both the general public and healthcare professionals. The campaign further encouraged lay people to get involved in their own health through self-recognition of sleep patterns. It was felt that such an initiative would facilitate professional care. For healthcare professionals, the aim was to improve their understanding of this new area and develop better diagnostic tools for recognizing and treating different sleep disorders.

We believe that the gradual growth in knowledge of Sleep Medicine will lead to an

Table 1. Outreach initiatives and estimated audience of the Sleep Medicine Program (2001-2004).

\begin{tabular}{lcr} 
Outreach vehicle & Quantity & Estimated average audience \\
\hline Media & & \\
$\quad$ Newspapers & 53 & 225,000 \\
Magazines & 51 & 229,000 \\
$\quad$ Radio & 21 & 45,000 \\
$\quad$ Television & 65 & $3,974,000$ \\
Others & 1 & 400,000 \\
$\quad$ Website & 2 & 41,000 \\
Exhibitions & 7 & 1,187 \\
Courses/lectures & 2 & 50,000 \\
$\quad$ Distribution of inserts on sleep & & \\
\hline
\end{tabular}

increased awareness regarding sleep disorders and ultimately will improve recognition and treatment options in public and private healthcare organizations.

The present study was designed to evaluate the influence of a Sleep Medicine outreach program on polysomnography (PSG) referrals to the Sleep Institute.

\section{Material and Methods}

\section{Sleep Medicine Outreach Program}

The outreach program was developed in January 2001 and was created in order to enhance awareness of sleep among the general public and healthcare professionals. Topics included sleep physiology, sleep hygiene, sleep rhythms, shift workers, accidents due to sleep problems, as well as review of various sleep disorders.

The program was divided into the following activities: organization of artistic exhibits with information about sleep, elaboration of the WEB page of the CEPID Center for Sleep Studies (www.sono.org.br), talks, lectures and sleep information classes, distribution of fliers on sleep, publication of scientific articles on sleep, and dissemination of sleep information through interviews with different professionals trained in sleep medicine (television, radio, newspapers, and magazines).

The outreach activities from January 2001 to December 2004 were organized according to the type of public to which it was directed (general public/healthcare professionals; Tables 1 and 2).

\section{Patient data}

A total of 8805 patients referred to the Sleep Institute from February to December 2000 and from January to December 2004 were analyzed. All patients were referred by physicians who were not associated with the Sleep Institute. The results of sleep studies 
were sent to the referring or primary physician who was responsible for decisions concerning treatment.

Based on diagnostic hypotheses highlighted by medical referrals, patients were classified into 8 groups of referral type:

Sleep-disorder breathing (SDB). OSAHS, snoring, upper airway resistance syndrome, and other otorhinolaryngological diseases such as deviated septum, turbinate hypertrophy, tonsil hypertrophy, and rhinitis.

Treatment of OSAHS (OSAHS- $t$ ). OSAHS patients treated with positive airway pressure or oral appliances.

Post-surgical OSAHS (OSAHS-ps). Follow-up of surgical treatment of OSAHS.

Parasomnia. Sleepwalking, sleeptalking, bruxism, night terror, REM behavior disorder, night enuresis, nightmare, sleep paralysis, hypnagogic hallucination.

Excessive daytime sleepiness (EDS). EDS, tiredness, fatigue, asthenia, residual sleepiness, hypersomnolence.

Insomnia. Insomnia, early awakening, difficulty in beginning or maintaining sleep, non-restorative sleep, sleep misperception.

Limb movement disturbance (LMD). LMD, limb movements, restless legs syndrome, periodic limb movement, myoclonia.

Non-specified. PSG referral without diagnostic hypotheses or reasons.

In order to identify the effects of the outreach program on PSG referrals to the Sleep Institute, the following parameters were evaluated and compared between the year prior to the program onset (February to December 2000) and the 4th year of the program (January to December 2004): number of beds for PSGs, number of PSGs performed, number of referrals according to different areas in São Paulo State (the state capital, Greater São Paulo, interior areas/ coastal region of the state), characteristics of patient profile and referral type, prevalence, and correspondence between referral type and objective PSG parameters. The follow- ing criteria were used for SDB identification: apnea and hypopnea index (AHI) of 5 events or more per sleep hour (19) and AHI $\geq 10$. The latter criterion was adopted in order to have more restrictive criteria of OSAHS (20). For LMD, index of periodic limb movement (PLMI) greater than 5 events per hour of sleep (21); for the referral type of insomnia, EDS and parasomnia we analyzed the association of different referral types by the same physician in 2000 and 2004. The reasoning behind this decision is that clinical symptoms are also necessary for a final diagnosis of this sleep disorders.

In order to analyze the effects of the outreach program on other sleep laboratories we determined the increase in number of PSG beds from 2000 to 2004 for a sample of 40 sleep laboratories in the north, south, southeast, and center-west regions of Brazil. This sample was derived from a list of 97 sleep laboratories registered with the Brazilian Sleep Society.

\section{Statistical analysis}

Differences in qualitative variables expressed in frequency and percentage terms were calculated using the chi-square $\left(\chi^{2}\right)$ test. To compare quantitative variables, reported as means $\pm \mathrm{SD}$, we used the Student $t$ test for independent samples. The level of significance was set at $\mathrm{P}<0.05$ in all analyses. Statistical analyses were performed using the Statistic software (version 5.1; StatSoft, Inc.; 1997).

Table 2. Initiatives to reach healthcare professionals (2001-2004).

Outreach vehicle

Quantity Professionals reached

Polysomnography course

Postgraduate specialization in Sleep Medicine

Distribution of inserts/articles in magazines for

healthcare professionals

Lectures and talks/classes

$8 \quad 337$

$5 \quad 52$

$3 \quad 55,000$

60

5,232 


\section{Results}

From 2000 to 2004, the number of PSG beds at the Sleep Institute increased by $52 \%$ (25 vs 38) and there were a total of 8805 incoming PSG referrals (2000: $24.6 \%$ vs 2004: 75.4\%; $\mathrm{P}<0.001$ ).

Residents in the State of São Paulo accounted for $97.2 \%$ of the patients $(2000, \mathrm{~N}=$ $2110 ; 2004, \mathrm{~N}=6452$ ), most of them from the state capital $(69.6 \%$; $\mathrm{P}<0.001)$. Although there was no significant difference between 2000 and 2004 in the proportion of patients referred from the capital and greater São Paulo, in 2004 there were proportionally fewer patients from the coastal or interior regions of the state in comparison to 2000 (Table 3).

In comparison to 2000 , more women were referred in 2004 (31.1 vs 37.2\%; $\mathrm{P}<$ 0.001 ), mainly with the following referral type: non-specified (27.9 vs 39.7\%; $\mathrm{P}<$ $0.001)$, SDB (31.7 vs 35.2\%; $\mathrm{P}<0.05)$, OSAHS-t (16.0 vs 27.0\%; $\mathrm{P}=0.003)$, and

\begin{tabular}{|c|c|c|c|}
\hline & 2000 & 2004 & $\begin{array}{c}\text { Total } \\
\text { (2000 and 2004) }\end{array}$ \\
\hline $\mathrm{N}$ & 2164 & 6641 & 8805 \\
\hline Age $($ mean \pm SD) & $47.4 \pm 15.5$ & $47.1 \pm 13.2$ & $47.2 \pm 13.8$ \\
\hline Referrals from São Paulo (N) & 2110 & 6452 & 8562 \\
\hline São Paulo city & $69.4 \%(1464)$ & $69.2 \%(4491)$ & $69.6 \%(5955)$ \\
\hline Greater São Paulo & $19.0 \%(401)$ & $20.6 \%(1327)$ & $20.2 \%(1728)$ \\
\hline Coast/interior & $11.6 \%(245)$ & $9.8 \%(634)^{*}$ & $10.3 \%(879)$ \\
\hline Referral type $(\mathrm{N})$ & 1951 & 6522 & 8473 \\
\hline Not specified & $26.9 \%(524)$ & $21.2 \%(1382)^{*}$ & $22.5 \%(1906)$ \\
\hline SDB & $54.7 \%(1068)$ & $54.3 \%(3541)$ & $54.4 \%(4609)$ \\
\hline OSAHS-t & $8.3 \%(162)$ & $12.3 \%(800)^{\star}$ & $11.4 \%(962)$ \\
\hline OSAHS-ps & $2.3 \%(44)$ & $1.6 \%(103)^{*}$ & $1.7 \%(147)$ \\
\hline Insomnia & $3.5 \%(69)$ & $6.5 \%(426)^{*}$ & $5.8 \%(495)$ \\
\hline EDS & $4.3 \%(83)$ & $4.2 \%(275)$ & $4.2 \%(358)$ \\
\hline LMD & $1.5 \%(30)$ & $1.4 \%(91)$ & $1.4 \%(121)$ \\
\hline Parasomnia & $1.3 \%(26)$ & $1.8 \%(120)$ & $1.7 \%(146)$ \\
\hline
\end{tabular}

$\mathrm{SD}$ = standard deviation; SDB = sleep-breathing disorder; OSAHS = obstructive sleep apnea-hypopnea syndrome; OSAHS- $t=$ treatment for OSAHS with continuous positive airway pressure or oral appliances; OSAHS-ps = follow-up of surgical treatment of OSAHS; EDS = excessive daytime sleepiness; LMD = limb movement disturbance. ${ }^{*} \mathrm{P}<0.05$ compared to year 2000 ( $\chi^{2}$ test).
OSAHS-ps $(13.6$ vs 32.0\%; $\mathrm{P}<0.05)$. The average age of referred women was significantly higher than that of men in 2000 and 2004 (women: $49.3 \pm 13.3$ vs men: $46.0 \pm$ 14.0 years; $\mathrm{P}<0.001)$.

$\mathrm{SDB}$ was the main reason for referrals in both periods. Non-specified and OSAHS-ps referrals declined over the 2000-2004 period. The referrals for OSAHS-t and insomnia increased in frequency in 2004 (Table 3).

In the group of patients referred for SDB, $72.7 \%$ pointed out $\mathrm{AHI} \geq 5$ in 2000 and $67.7 \%$ in $2004(\mathrm{P}=0.004)$. Regarding AHI $\geq 10$, the percentage of patients for all years taken together was $51 \%$.

The prevalence of $\mathrm{AHI} \geq 5$ in referral types not related to SDB, OSAHS-t, or OSAHS-ps and with registered AHI was: non-specified, $65.9 \%$ (1202); LMD, 54.4\% (68); parasomnia, $47.9 \%$ (35), and there was no statistically significant difference across years for such referral types. However, in comparison to 2000, the prevalence of AHI $\geq 5$ increased in 2004 for insomnia referral types (36.2 vs 53.5\%, $\mathrm{P}=0.03)$ and $\mathrm{EDS}$ (33.3 vs $58.7 \%, \mathrm{P}=0.03$ ).

Referrals for LMD with PLMI registered $(\mathrm{N}=52)$ showed $36.5 \%$ PLMI $\geq 5$, with no significant difference over the years. The prevalence of PLMI $\geq 5$ in referrals not related to LMD and with registered PLMI was statistically equivalent across the years to referrals for insomnia [21.4\% (40)] and parasomnia $[21.8 \%(12)]$. For the other referral types, this prevalence decreased in 2004: non-specified [2000: $47.7 \%$ (146) vs 2004: $21.0 \%$ (95)], SDB [2000: $43.3 \%$ (256) vs 2004: $19.9 \%$ (264)] and EDS [2000: $40.9 \%$ (18) vs 2004: $15.8 \%$ (15)], with $\mathrm{P}<0.001$ for all analyses.

Referrals for insomnia were more frequently found together with SDB, OSAHSt or OSAHS-ps on the same referrals in 2004 (2000: $14.5 \%$ vs 2004: $28.9 \%$; $\mathrm{P}=0.01$ ) but were less frequent with LMD (2000: $13.0 \%$ vs 2004: $2.8 \% ; \mathrm{P}=0.003$ ) or the EDS referral type (2000: $11.6 \%$ vs 2004: $5.2 \%$; $\mathrm{P}=$ 
0.04). The EDS referral type was less associated with insomnia referral type in 2004 (2000: $30.0 \%$ vs 2004: $13.2 \%$; $\mathrm{P}=0.03$ ).

In the surveyed sleep laboratories located in different regions of Brazil, PSG beds increased by $92.7 \%$ during the 2000 2004 period (96 vs 185).

\section{Discussion}

The increase in the number of PSG beds in 2000-2004 corresponded to an increase in external referrals for PSG. We posit that this was linked to an overall better awareness of Sleep Medicine by health care professionals and the public at large. The relatively lower number of patients from the interior or coastal regions of the state registered in 2004 may suggest that a significant number of sleep laboratories were opened in these regions, absorbing the drop in population registered. The substantial increase in the number of beds for PSG over the 2000-2004 period in different regions of Brazil (92.7\%) supports this claim and reflects the more widespread knowledge of Sleep Medicine, especially among physicians.

In addition to the increase in number of beds and referrals, the quality of the information conveyed was also evaluated as an important factor related to the ensemble of outreach initiatives. Schillinger et al. (17) found that sleep awareness programs led to family doctors reporting positive changes in their clinical practice: better anamnesis with more attention to sleep problems and more confidence in recognizing, diagnosing and treating sleep disorders. The present study analyzed the relationship between the initiatives in sleep education and their possible impact on clinical practice, in particular regarding the diagnostic hypothesis shown in medical referrals. The findings showed a significant decrease in the proportion of nonspecified referral type in 2004, probably reflecting greater confidence in clinical practice across the years.
Since menopause is a risk factor for sleep apnea in women $(22,23)$ and given that PSG is considered to be an important exam for the diagnosis of SDB (19), the increase in the proportion of women referred between 2000 and 2004, in particular for SDB, OSAHS-t and OSAHS-ps, with mean ages similar to those described in the literature for menopausal women (24), suggests an improvement of medical knowledge surrounding the association of SDB linked to gender.

As shown in another study (25), SDB referral type was also the main reason for referring patients for PSG and included the following diagnostic hypotheses in our population: snoring, upper airway resistance syndrome, otorhinolaryngological diseases, and OSAHS. The treatment of OSAHS can be mechanical (positive airway pressure or oral appliances) or surgical. The best available treatment for OSAHS is positive airway pressure (26). Oral appliances are indicated for patients with mild OSAHS or those who refuse or do not tolerate positive airway pressure (27). The surgical alternative is more restricted and the results of maxillomandibular advanced surgery have been more effective than surgery on airway soft tissue (28). The surgical outcome is favored by factors such as the site of airway obstruction, lower body mass index and report of less severe OSAHS (29). Moreover, surgery may be an option when positive airway pressure is indicated, but when the patient is unable to adapt to the device (30). The present study highlighted an increase in the number of referrals for OSAHS-t and a decrease in referrals for OSAHS-ps in the 2000-2004 period. This led us to single out improved training among physicians for the medical treatment of SDB.

The agreement index between SDB referral type and AHI $\geq 5$ was high for both periods analyzed, suggesting that physicians were more concerned about basic knowledge of SDB, as also shown in another study (31). However, the decrease of the agreement index across the 4 years of the study 
may point out that there is still a need for more enhanced awareness of sleep medicine beyond our outreach program.

When an AHI $\geq 10$ was adopted as a criterion for analysis, the SDB referral type showed a lower incidence than that reported by Jonczak et al. (20) (52.1 vs 58\%, respectively). This may be explained by the fact that Jonczak et al. (20) analyzed only referrals involving a direct suspicion of OSAHS, while our study looked at SDB.

The prevalence of AHI $\geq 5$ among nonspecified referrals was comparable to that among patients referred for SDB evaluation. It is possible that some physicians do not have adequate training to recognize SDB symptoms and to ask specific questions regarding this condition.

The increase of the prevalence rate of AHI $\geq 5$ among EDS referral types across the years may be a consequence of greater awareness among physicians about sleepiness as a symptom of sleep apnea (19). This is supported by a lower association between EDS and insomnia referral type in the same medical referral found in 2004 compared to 2000.

Recent literature reports have claimed that more labored breathing in upper airway resistance syndromes seems to evoke or facilitate the emergence of limb movement disturbance (32). However, the higher prevalence of $\mathrm{AHI} \geq 5$ in the LMD referral type cannot be explained as a consequence of heightened awareness among physicians. It was more clearly identified in insomnia referral type. In 2004, referrals for insomnia were more associated with SDB and less with LMD or EDS referral type. Moreover, in 2004 insomnia referral type showed a significant increase of the prevalence of AHI $\geq 5$. PSG referral based on insomnia referral type seems to indicate that physicians are more concerned to seek out a cause for insomnia before asserting that the patient's complaint is primary insomnia. Additionally, our results show more clinical emphasis on the relation between insomnia and
SDB, as reported in the literature (33-35). Some patients who were referred to centers because of insomnia complaint may have what is described as a syndrome of "hidden" sleep apnea (36), in which night awakenings and the tendency for sleep to become more superficial lead to complaints of insomnia, and when the aggravation of OSAHS leads to decreased somatic syndrome, including insomnia $(33,35)$.

The difficulty in differentiating diagnoses of insomnia and SDB points to the absence of effective clinical strategies for the diagnosis of insomnia (37) and the need for an interdisciplinary approach to the treatment of sleep disorders (38). Moreover, it suggests that, although some studies do not indicate PSG for routine evaluation of transitory or chronic insomnia and insomnia associated with psychiatric disturbances (37, 39), PSG is still an indispensable resource for the differential diagnosis of insomnia and SDB or other sleep disorders.

The prevalence of PLMI $\geq 5$ in unspecified referral types, SDB and EDS decreased over the period from 2000 to 2004, supporting the suggestion that physicians have become more assertive concerning the definition of the diagnostic hypotheses related to sleep disorders.

We conclude that there is a need to develop tools for evaluating sleep and the use of PSG as an instrument to assist in differentiating sleep disorders. Moreover, the present study demonstrated that Sleep Medicine awareness increased over the 4 years of our outreach program, reflecting more assertive referrals. This result highlights the importance of the outreach initiatives in Sleep Medicine in a country's health care system. However, regarding Sleep Medicine education, it is still necessary to offer a differentiated mechanism through a comprehensive approach that encompasses sleep disorders in general. The most effective strategy for this is to restructure and reevaluate syllabuses for courses in medicine and related fields. 


\section{References}

1. Young T, Palta M, Dempsey J, Skatrud J, Weber S, Badr S. The occurrence of sleep-disordered breathing among middle-aged adults. N Engl J Med 1993; 328: 1230-1235.

2. Ford DE, Kamerow DB. Epidemiologic study of sleep disturbances and psychiatric disorders. An opportunity for prevention? JAMA 1989; 262: 1479-1484.

3. Mellinger GD, Balter MB, Uhlenhuth $\mathrm{EH}$. Insomnia and its treatment. Prevalence and correlates. Arch Gen Psychiatry 1985; 42: 225-232.

4. National Commission on Sleep Disorders Research. Wake Up America: a national sleep alert. Washington: Government Printing Office; 1993.

5. Phillipson EA. Sleep apnea - a major public health problem. $N$ Engl J Med 1993; 328: 1271-1273.

6. Bagnato MC, Togeiro SM, Bittencourt LRA. The prevalence of snoring in São Paulo City, Brazil. J Sleep Res 1996; 5 (Suppl 1): 11.

7. Rosen RC, Zozula R, Jahn EG, Carson JL. Low rates of recognition of sleep disorders in primary care: comparison of a communitybased versus clinical academic setting. Sleep Med 2001; 2: 47-55.

8. Meissner HH, Riemer A, Santiago SM, Stein M, Goldman MD, Williams AJ. Failure of physician documentation of sleep complaints in hospitalized patients. West J Med 1998; 169: 146-149.

9. Rosen RC, Rosekind M, Rosevear C, Cole WE, Dement WC. Physician education in sleep and sleep disorders: a national survey of U.S. medical schools. Sleep 1993; 16: 249-254.

10. Zozula R, Bodow M, Yatcilla D, Cody R, Rosen RC. Development of a brief, self-administered instrument for assessing sleep knowledge in medical education: "the ASKME Survey". Sleep 2001; 24: $227-$ 233.

11. Lavie P. Physician education in sleep disorders - a dean of medicine's viewpoint. Sleep 1993; 16: 760-761.

12. Strohl KP, Veasey S, Harding S, Skatrud J, Berger HA, Papp KK, et al. Competency-based goals for sleep and chronobiology in undergraduate medical education. Sleep 2003; 26: 333-336.

13. Federman DD. Competency-based goals for sleep and chronobiology in undergraduate medical education. Sleep 2003; 26: 251.

14. Rosen R, Mahowald M, Chesson A, Doghramji K, Goldberg R, Moline M, et al. The Taskforce 2000 survey on medical education in sleep and sleep disorders. Sleep 1998; 21: 235-238.

15. Stores G, Crawford C. Medical student education in sleep and its disorders. J R Coll Physicians Lond 1998; 32: 149-153.

16. Orr WC, Stahl ML, Dement WC, Reddington D. Physician education in sleep disorders. J Med Educ 1980; 55: 367-369.

17. Schillinger E, Kushida C, Fahrenbach R, Dement W, LeBaron S. Teaching family medicine medical students about sleep disorders. Fam Med 2003; 35: 547-549.

18. Mahendran R, Subramaniam M, Chan YH. Medical students' behaviour, attitudes and knowledge of sleep medicine. Singapore Med $J$ 2004; 45: 587-589.

19. American Academy of Sleep Medicine. Sleep-related breathing disorders in adults: recommendations for syndrome definitions techniques in clinical research. Sleep 1999; 22: 667-689.

20. Jonczak L, Sliwinski P, Cieslicki J, Koziej M, Mankowski M, Plywaczewski R, et al. Ten years experience of the sleep laboratory at the Institute of Tuberculosis and Lung Disease in Warsaw. Pneumonol Alergol Pol 2001; 69: 538-544.
21. American Sleep Disorders Association. Practice parameters for the treatment of restless leg syndrome and periodic limb movement disorders. Sleep 1999; 22: 968.

22. Dancey DR, Hanly PJ, Soong C, Lee B, Hoffstein V. Impact of menopause on the prevalence and severity of sleep apnea. Chest 2001; 120: 151-155.

23. Bixler EO, Vgontzas AN, Lin HM, Ten HT, Rein J, Vela-Bueno A, et al. Prevalence of sleep-disordered breathing in women: effects of gender. Am J Respir Crit Care Med 2001; 163: 608-613.

24. Young T, Finn L, Austin D, Peterson A. Menopausal status and sleep-disordered breathing in the Wisconsin Sleep Cohort Study. Am J Respir Crit Care Med 2003; 167: 1181-1185.

25. Hoffstein V. Relationship between smoking and sleep apnea in clinic population. Sleep 2002; 25: 519-524.

26. Heitman SJ, Flemons WW. Evidence-based medicine and sleep apnea. Respir Care 2001; 46: 1418-1432.

27. Dal Fabbro C, Bittencourt L, Tufik S. The effect of an oral appliance (Kleanway) on obstructive sleep apnea and hypopnea syndrome (OSAHS). Sleep 2001; 24: A280-A282 (Abstract).

28. Prinsele JR. Maxillomandibular advanced surgery for obstructive sleep apnea syndrome. J Am Dent Assoc 2002; 133: 1489-1497.

29. Sher AE, Schechtman KB, Piccirillo JF. The efficacy of surgical modifications of the upper airway in adults with obstructive sleep apnea syndrome. Sleep 1996; 19: 156-177.

30. Souter MA, Stevenson S, Sparks B, Drennan C. Upper airway surgery benefits patients with obstructive sleep apnoea who cannot tolerate nasal continuous positive airway pressure. J Laryngol Otol 2004; 118: 270-274.

31. Phillips B, Collop N, Goldberg R. Sleep medicine practices, training, and attitudes: a wake-up call for pulmonologists. Chest 2000; 117 : 1603-1607.

32. Exar EN, Collop NA. The association of upper airway resistance with periodic limb movements. Sleep 2001; 24: 188-192.

33. Gold AR, Dipalo F, Gold MS, O'Hearn D. The symptoms and signs of upper airway resistance syndrome: a link to the functional somatic syndromes. Chest 2003; 123: 87-95.

34. Krakow B, Melendrez D, Ferreira E, Clark J, Warner TD, Sisley B, et al. Prevalence of insomnia symptoms in patients with sleep-disordered breathing. Chest 2001; 120: 1923-1929.

35. Chung KF. Relationships between insomnia and sleep-disordered breathing. Chest 2003; 123: 310-311.

36. Collop NA. Can't sleep? You may have sleep apnea $\chi$ Chest 2001; 120: 1768-1769.

37. Chesson A Jr, Hartse K, Anderson WM, Davila D, Johnson S, Littner $\mathrm{M}$, et al. Practice parameters for the evaluation of chronic insomnia. An American Academy of Sleep Medicine report. Standards of Practice Committee of the American Academy of Sleep Medicine. Sleep 2000; 23: 237-241.

38. Krakow B. An emerging interdisciplinary sleep medicine perspective on the high prevalence of co-morbid sleep-disordered breathing and insomnia. Sleep Med 2004; 5: 431-433.

39. Littner M, Hirshkowitz M, Kramer M, Kapen S, Anderson WM, Bailey $D$, et al. Practice parameters for using polysomnography to evaluate insomnia: an update. Sleep 2003; 26: 754-760. 